Reprod. Nutr. Dévelop., 1985, 25 (1 B), 321-327.

\title{
Evolution des dépenses énergétiques du rat Zucker au cours de la première semaine de la vie
}

\author{
Eléonore PLANCHE, M. JOLIFF
}

Laboratoire sur la Physiopathologie de la Nutrition, INSERM, U.177, Institut Biomédical des Cordeliers, 15, rue de l'Ecole de Médecine, 75006 Paris, France.

Summary. Changes in energy expenditure in the Zucker rat during the first neonatal week.

The respiratory $\mathrm{CO}_{2}$ production and $\mathrm{O}_{2}$ consumption of Zucker fa/fa and $\mathrm{Fa} / \mathrm{fa}$ rats 2, 5 and 7 days old were measured for two hours from 16.30 to $18.30 \mathrm{~h}$. Metabolic studies were performed at five different temperatures : $26,28,30,33$ and $35{ }^{\circ} \mathrm{C}$. At all the ages studied, maximal energy expenditure was observed at an ambient temperature of $30^{\circ} \mathrm{C}$. From the age of 2 days and for all the temperatures studied, except $35^{\circ} \mathrm{C}$ (thermoneutral temperature), the level of gaseous exchange was statistically lower in $\mathrm{fa} / \mathrm{fa}$ than in $\mathrm{Fa} / \mathrm{fa}$ pups. The difference between the two genotypes increased with age. On the other hand, there was no difference in the level of the respiratory quotient between the two genotypes, whatever the age or the temperature. In conclusion, the deficit in energy expenditure of the obese pups, that seemed to be related to a defective thermoregulatory thermogenesis, began very early and was probably a primary manifestation of their genetic defect. It was more than enough to account for their excess fat storage.

\section{Introduction.}

Depuis une dizaine d'années l'accent a été mis sur le rôle d'un déficit des dépenses énergétiques, lié à un défaut de la thermogenèse, dans le développement de l'obésité chez l'homme (Jéquier, 1983) et dans certains modèles d'obésité génétique (Boissonneault et al., 1978 ; Godbole et al., 1978 ; Trayhurn, 1979 ; James et Trayhurn, 1981). Dans un précédent travail (Planche et al., 1983), nous-mêmes avons montré que, à l'âge de 7 jours, le rat Zucker obèse $(\mathrm{fa} / \mathrm{fa})$ présentait effectivement, comparé au témoin hétérozygote $(\mathrm{Fa} / \mathrm{fa})$, un déficit de ses dépenses énergétiques qui portait à la fois sur la consommation $d^{\prime} \mathrm{O}_{2}$ et l'excrétion du $\mathrm{CO}_{2}$. Le travail actuel a été entrepris afin d'essayer de déterminer la chronologie d'installation de ces anomalies et de voir si leur évolution au cours de la $1^{\text {re }}$ semaine de la vie pouvait rendre compte de l'excès de lipides corporels présents, en l'absence d'hyperphagie, chez le futur obèse à l'âge de 7 jours (Boulangé et al., 1979). 


\section{Matériel et Méthodes.}

Animaux. - Les animaux utilisés étaient des rats Zucker obèses $(\mathrm{fa} / \mathrm{fa})$ et non obèses $(\mathrm{Fa} / \mathrm{fa})$ âgés de 2,5 et 7 jours, nés du croisement d'un mâle obèse et d'une femelle hétérozygote. Les petits et leur mère étaient conservés dans une animalerie dont la température était $22^{\circ} \pm 1{ }^{\circ} \mathrm{C}$, éclairée de $7 \mathrm{~h}$ à $19 \mathrm{~h}$. Les animaux étaient étudiés à l'état nourri. Après les mesures, les ratons étaient pesés, marqués et rendus à leur mère. Le génotype était identifié à l'âge de 6 semaines.

Echanges gazeux. - Les échanges gazeux étaient mesurés dans un appareil à circuit fermé permettant la mesure conjointe de $\mathrm{I}^{\prime} \mathrm{O}_{2}$ consommé et du $\mathrm{CO}_{2}$ excrété comme préalablement décrit (Planche et al., 1983). Tous les petits d'une même portée (8-10 par portée) étaient étudiés simultanément. L'oxygène consommé était mesuré par lecture directe sur un réservoir gradué et corrigé pour la température et la pression atmosphérique (STPD). Le $\mathrm{CO}_{2}$ excrété était collecté dans un tube contenant $8 \mathrm{ml}$ de $\mathrm{NaOH} 1 \mathrm{~N}$ et mesuré par titrimétrie. Les résultats étaient exprimés en $\mathrm{ml} \mathrm{d} \mathrm{d}_{2}$ et de $\mathrm{CO}_{2}$ par $100 \mathrm{~g}$ de poids corporel.

Les mesures étaient effectuées pendant $2 \mathrm{~h}$, de $16 \mathrm{~h} 30$ à $18 \mathrm{~h} 30$. L'heure de la mesure avait été déterminée par une expérience préliminaire portant sur l'excrétion du $\mathrm{CO}_{2}$ de rats de 7 jours au cours de la période diurne. La figure 1

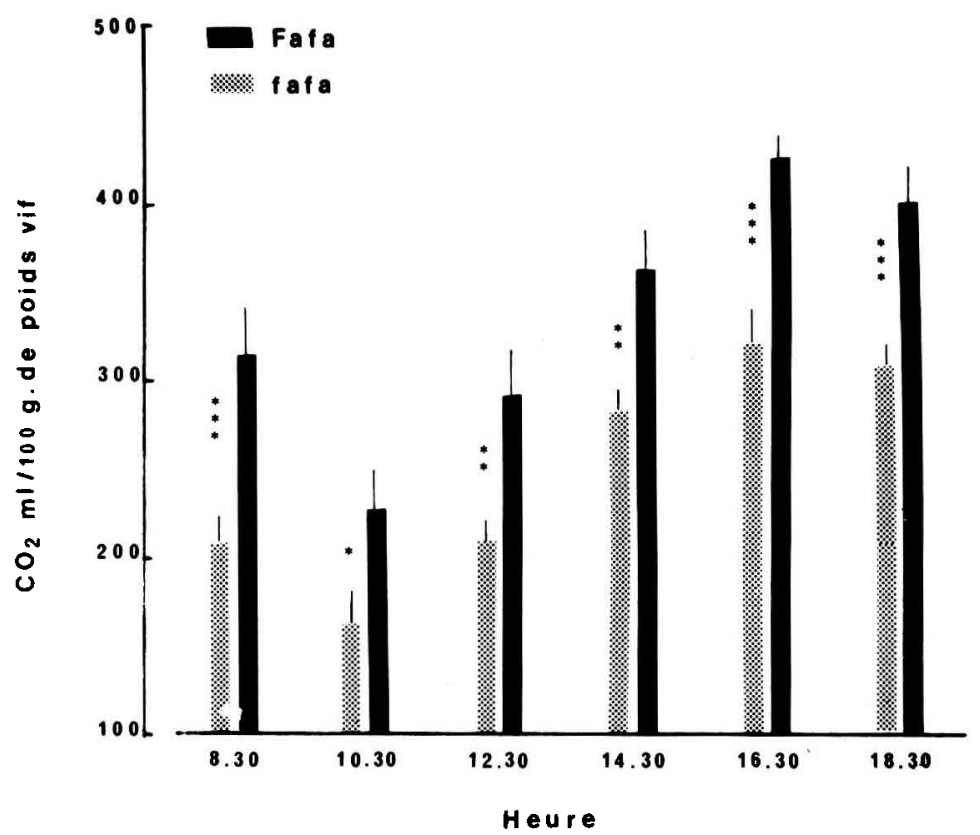

FIG. 1. - Evolution de l'excrétion du $\mathrm{CO}_{2}$ au cours de la période diurne.

L'excrétion du $\mathrm{CO}_{2}$ était mesurée pendant $2 \mathrm{~h}$ toutes les $2 \mathrm{~h}$ de $8 \mathrm{~h} 30$ à $20 \mathrm{~h} 30$ à la température ambiante de $28{ }^{\circ} \mathrm{C}$ sur des rats fa/fa (N:97) et $\mathrm{Fa} / \mathrm{fa}(\mathrm{N}: 60)$ àgés de 7 jours et pesant respectivement $11,9 \pm 0,2 \mathrm{~g}$ et $12,0 \pm 0,2 \mathrm{~g}$.

${ }^{*}: \mathrm{P}<0,05 ; ;^{* *}: \mathrm{P}<0,01 ;{ }^{* * *}: \mathrm{P}<0,001$. 
montre que le maximum des dépenses dans les 2 groupes d'animaux et le maximum de différence entre les 2 génotypes se situaient à cette période de la journée. Les animaux étaient étudiés à 5 températures ambiantes : $35^{\circ} \mathrm{C}$ (température de neutralité thermique), $33^{\circ}, 30^{\circ}, 28^{\circ}$ et $26^{\circ} \mathrm{C}$ : températures tenant compte de celles de l'environnement habituel des rats de cet âge (tabl. 1 ).

TABLEAU 1

Evolution de la température du nid en l'absence de la mère (*).

\begin{tabular}{|c|c|c|c|c|c|}
\hline & \multirow{2}{*}{$\begin{array}{l}0 \text { minute } \\
\text { mère sur le nid }\end{array}$} & \multicolumn{4}{|c|}{ Temps d'absence de la mère (minutes) } \\
\hline & & 15 & 30 & 45 & 60 \\
\hline Température du nid ${ }^{\circ} \mathrm{C}$ & $33,2 \pm 0,2$ & $29,6 \pm 0,6$ & $28,3 \pm 0,5$ & $27,1 \pm 0,4$ & $24,9 \pm 0,6$ \\
\hline
\end{tabular}

Statistiques. - Les résultats étaient exprimés en moyenne $\pm S E$. Les comparaisons entre les 2 génotypes étaient faites en utilisant le test t de Student.

\section{Résultats.}

Poids des animaux (tabl. 2). - Ce tableau montre qu'il n'existe pas, aux âges étudiés, de différence dans le poids corporel, entre les 2 génotypes.

TABLEAU 2

Poids des rats $(g)$.

\begin{tabular}{ccc}
\hline & $\mathrm{fa} / \mathrm{fa}$ & $\mathrm{Fa} / \mathrm{fa}$ \\
\hline $2 \mathrm{~J}$ & $\begin{array}{c}7,38 \pm 0,06 \\
(64)\end{array}$ & $\begin{array}{c}7,41 \pm 0,06 \\
(59)\end{array}$ \\
\hline $5 \mathrm{~J}$ & $\begin{array}{c}10,37 \pm 0,07 \\
(63)\end{array}$ & $\begin{array}{c}0,36 \pm 0,08 \\
(47)\end{array}$ \\
\hline $7 \mathrm{~J}$ & $12,82^{ \pm \pm} 0,18$ & $12,47 \pm 0,14$ \\
$(60)$ & $(46)$ \\
\hline
\end{tabular}

Echanges gazeux (fig. 2). - A $35{ }^{\circ} \mathrm{C}$ le niveau des échanges gazeux était très bas et on n'observait pas de différence entre les 2 génotypes. A tous les âges étudiés et dans les 2 groupes d'animaux, le niveau des échanges gazeux augmentait significativement $(P<0,001)$ dès que la température ambiante était abaissée, le maximum était atteint à $30^{\circ} \mathrm{C}$. Cependant les obèses augmentaient moins leurs dépenses que les témoins de sorte que la différence entre eux était significative à toutes les températures étudiées. Cette différence augmentait avec 


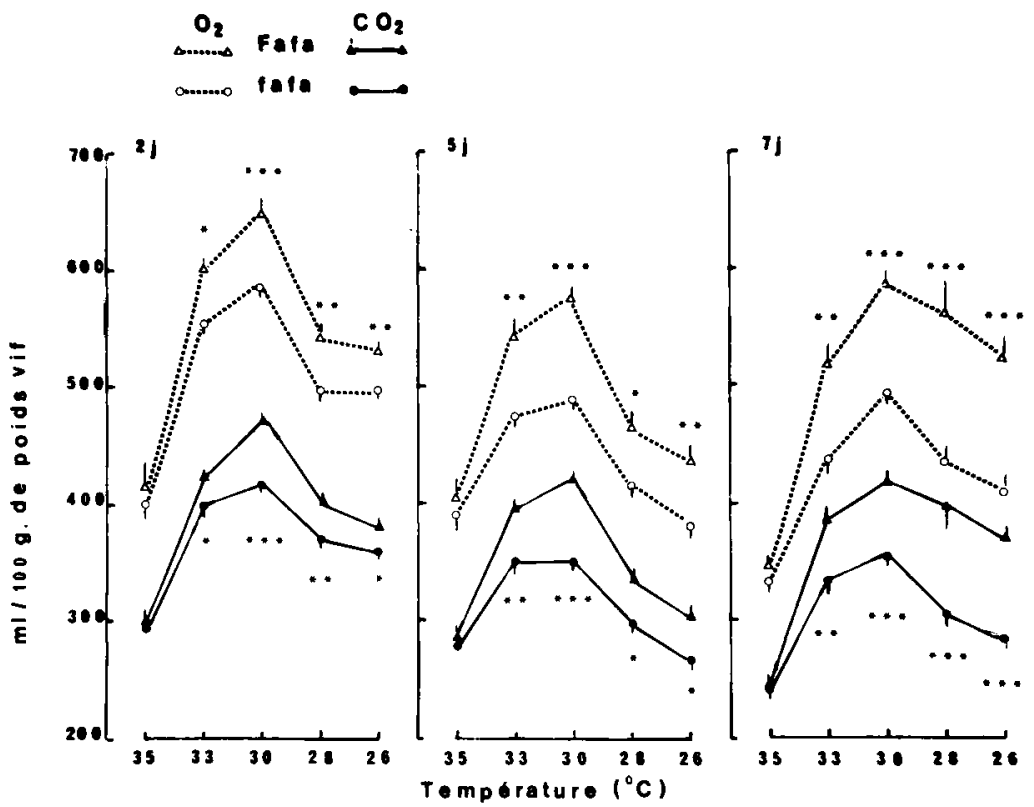

FIG. 2. - Evolution des échanges gazeux en fonction de l'âge et de la température ambiante. Les échanges gazeux étaient mesurés pendant $2 \mathrm{~h}$ chez des rats Zucker âgés de 2,5 et 7 jours à 5 températures différentes.

Le nombre et le poids des animaux sont donnés dans le tableau 1.

Les symboles sont identiques à ceux de la figure 1 .

I'âge des animaux. Entre $30^{\circ}$ et $28^{\circ}$ on observait dans tous les groupes une diminution du niveau des échanges gazeux. Cette diminution était très significative à 2 jours et à 5 jours $(P<0,001)$ dans les 2 génotypes et à 7 jours chez les $\mathrm{fa} / \mathrm{fa}(\mathrm{P}<0,01)$ mais pas chez les $\mathrm{Fa} / \mathrm{fa}$, de sorte qu'à cet âge c'est à la température de $28^{\circ}$ que la différence entre les 2 groupes d'animaux était la plus importante. Entre $28^{\circ}$ et $26^{\circ}$ le niveau des échanges gazeux diminuait légèrement d'une manière comparable quels que soient l'âge et le génotype des animaux.

\section{Discussion.}

Bien que l'homéothermie ne soit pas totalement acquise chez le rat avant l'âge de 3-4 semaines (Conklin et Heggeness, 1971), le raton nouveau-né est cependant capable de lutter contre l'abaissement de la température ambiante, au moins dans certaines limites, grâce aux mécanismes de la thermogenèse sans frisson (Hahn et Novak, 1975), qui se traduisent au niveau fonctionnel par une augmentation des échanges gazeux (Fuller et al., 1983). Nos résultats montrent que ces mécanismes fonctionnaient chez le Zucker nouveau-né, au moins dès l'âge de 2 jours et quel que soit son génotype, encore que, sauf à $35^{\circ}$ (thermoneutralité), la réponse des obèses à l'abaissement de la température 
ambiante soit significativement plus faible que celle des témoins, même à 2 jours et même à $33^{\circ} \mathrm{C}$, température la plus chaude de leur environnement habituel. Cependant la capacité thermogénétique des ratons était rapidement dépassée puisque au-dessous du seuil de $30^{\circ} \mathrm{C}$, le niveau des échanges gazeux tendait à diminuer. A 7 jours cette diminution $n$ 'était significative $(P<0,01)$ que chez les $\mathrm{fa} / \mathrm{fa}$, la courbe de leur réponse au froid étant similaire à celle des 2 groupes d'animaux à 2 et 5 jours. Il semble donc que, non seulement, le futur obèse aurait une capacité thermogénétique diminuée mais encore qu'il présenterait un retard dans l'installation d'une relative homéothermie.

Un déficit précoce analogue a été décrit chez la souris ob/ob au cours de la $1^{\text {re }}$ semaine de la vie (Romsos et al., 1979). Ce déficit, comme dans notre étude, était lié à une anomalie de la réponse au froid, donc probablement de la thermogénèse sans frisson. Le $\mathrm{fa} / \mathrm{fa}$ adulte présente également un déficit de ses dépenses énergétiques, de plus il devient hypothermique lorsque la température ambiante est abaissée (Trayhurn et al., 1973), cependant le rôle d'une anomalie de la thermogenèse sans frisson dans le déficit énergétique du fa/fa adulte est controversé. Certains auteurs observent une diminution de la réponse thermogénique à la noradrénaline dans le tissu adipeux brun de l'obèse (Wickler et al., 1982), d'autres attribuent son déficit énergétique uniquement à une anomalie de la thermogenèse induite par l'alimentation (Rothwell et Stock 1983 ; Triandafillou et Himms-Hagen, 1983). Ce dernier type d'anomalie ne semble pas être présent chez le $\mathrm{fa} / \mathrm{fa}$ au cours de la 1 re semaine de la vie, puisque, bien que les animaux aient été étudiés à l'état nourri, à la température de neutralité thermique nous n'avons pas observé de différence entre les 2 génotypes. Quelle qu'en soit l'origine, le site principal de la thermogenèse est le tissu adipeux brun (Foster et Frydman, 1979). La production de chaleur est due à une augmentation de l'oxydation des substrats, notamment les acides gras, qui résulte du découplage des mitochondries de ce tissu (Heaton et Nicholls, 1976). Ce découplage peut être mis en évidence in vitro par la mesure de la liaison de nucléotides puriques (GDP) à un site spécifique de la membrane interne mitochondriale (protéine découplante). Nos résultats sont à rapprocher et confirment sur le plan fonctionnel les travaux de Bazin et al. (1984) qui montrent effectivement une anomalie de la liaison du GDP dans les mitochondries du tissu adipeux brun du jeune fa/fa dès l'âge de 2 jours.

La question était de savoir si le déficit énergétique que nous avons mesuré pouvait rendre compte de l'excès de stockage des lipides corporels $\mathrm{du} \mathrm{fa} / \mathrm{fa}$ à l'âge de 7 jours. Le calcul théorique de la quantité de lipides stockés en excès par les obèses en fonction du déficit de leur consommation $d^{\prime} \mathrm{O}_{2}$ est montré dans le tableau 3. Ce calcul est basé sur la valeur calorique de l'oxygène en fonction du QR. Rapportées à $24 \mathrm{~h}$ ces quantités de lipides sont considérables et probablement surestimées puisqu'à 7 jours l'excès de lipides corporels des obèses par rapport aux témoins est d'environ $100 \mathrm{mg}$ (Planche et al., 1983). En fait cette étude ponctuelle de $2 \mathrm{~h}$, pratiquée volontairement au moment où les 2 génotypes sont les plus différents (fig. 1) ne tient pas compte des variations de comportement de la mère et des petits au cours du nychtémère. Cependant ce 
TABLEAU 3

Calcul théorique des lipides stockés en excès par les fa/fa.

\begin{tabular}{lccccc}
\hline & & & \multicolumn{2}{c}{\begin{tabular}{c}
\multicolumn{2}{c}{ Lipides en excès } \\
$\mathrm{mg} / \mathrm{rat}$
\end{tabular}} \\
& Age & Q.R. & $\begin{array}{c}\mathrm{m} / \mathrm{h} \\
\mathrm{ml}\end{array}$ & $\mathrm{h}$ & $\mathrm{h} \mathrm{h}$ \\
\hline $33^{\circ}$ & $2 \mathrm{~J}$ & 0,70 & 2,0 & 1,0 & 24 \\
& $5 \mathrm{~J}$ & 0,74 & 3,3 & 1,5 & 36 \\
& $7 \mathrm{~J}$ & 0,77 & 5,0 & 2,1 & 50 \\
\hline $28^{\circ}+30^{\circ}$ & $2 \mathrm{~J}$ & 0,72 & 1,8 & 0,8 & 19 \\
& $5 \mathrm{~J}$ & 0,71 & 3,5 & 2,3 & 56 \\
& $7 \mathrm{~J}$ & 0,70 & 6,6 & 3,3 & 79 \\
\hline
\end{tabular}

Ce calcul est basé sur la valeur calorique de l' $\mathrm{O}_{2}$ épargné par les fa/fa, en fonction du Q.R.

calcul montre que le déficit que nous avons mesuré est largement suffisant pour rendre compte de l'excès de lipides corporels du futur obèse à l'âge de 7 jours.

En conclusion : L'ensemble de nos observations montre, dès l'âge de 2 jours, l'existence d'un déficit énergétique chez le fa/fa nouveau-né. La précocité de ce déficit, qui est lié à une anomalie des mécanismes de la thermogenèse sans frisson, permet de penser que ces anomalies sont une expression primaire de la tare génétique liée au gêne fa.

$10^{\circ}$ Réunion du groupe Développement I.N.R.A., Rennes, 9-10 mai 1984.

Remerciements. - Nous remercions vivement Mademoiselle V. Resve qui a assuré la dactylographie de cet article.

\section{Références}

BAZIN R., ETĖVE D., LAVAU M., 1984. Evidence for decreased GDP binding to brown adipose tissue mitochondria of obese Zucker (fa/fa) rat in the very first days of life. Biochem. $J$. (in press).

BOISSONNEAULT G. A., HORNSHUH M. J., SIMONS J. W., ROMSOS D. R., LEVEILLE G. Z., 1978. Oxygen consumption and body fat content of young lean and obese (ob/ob) mice. Proc. Soc. exp. Biol. Med., 157, 401-406.

BOULANGÉ A., PLANCHE E., de GASQUET, P., 1979. Onset of genetic obesity in the absence of hyperphagia during the first week of life in the Zucker rat $(\mathrm{fa} / \mathrm{fa})$. J. Lipid Res., 20, 857-864.

CONKLIN P., HEGGENESS F. W., 1971. Maturation of temperature homeostasis in the rat. Am. J. Physiol., 220, 333-336.

FOSTER D. O., FRYDMAN L., 1979. Tissue distribution of cold-induced thermogenesis in conscious warm or cold-acclimated rats reevaluated from changes in tissue blood flow : the dominant role of brown adipose tissue in the replacement of shivering by non shivering thermogenesis. Can. J. Physiol. Pharmacol., 57, 257-270.

FULLER H. L., DALE N. M., SMITH C. F., 1983. Comparison of heat production of chickens measured by energy balance and by gaseous exchange. $J$. Nutr., 113, 1403-1408. 
GOBDOLE V., YORK D. A., BLOXHAM D. P., 1978. Developmental changes in the fatty (fa/fa) rat: Evidence for defective thermogenesis preceding the hyperlipogenesis and hyperinsulinemia. Diabetologia, 15, 41-44.

HAHN P., NOVAK M., 1975. Development of brown and white adipose tissue. J. Lipid Res., 16, 79-91.

HEATON G. M., NICHOLS D. G., 1976. Hamster brown adipose tissue mitochondria. The role of fatty acids in the control of proton conductance in the inner membrane. Eur. J. Biochem., 67, 511-517.

JAMES W. P. T., TRAYHURN, P., 1981. Thermogenesis and obesity. Br. Med. Bull., 37, 43-48.

JEOUIER E., 1983. Does a thermogenic defect play a role in the pathogenesis of human obesity? Clin. Physiol., 3, 1-7.

PLANCHE E., JOLIFF M., de GASQUET P., LE LIEPVRE X., 1983. Evidence of a defect in energy expenditure in 7-day-old Zucker rat $(\mathrm{fa} / \mathrm{fa})$. Am. J. Physiol., 245, E 104-E 107.

ROMSOS D. R., HORNSHUH M. J., LEVEILLE G. A., 1979. Influence of acute thermal stress and maternal diet on metabolic rate of obese (ob/ob) and lean mice at two weeks of age. Int. J. Obesity, 3, 249-254.

ROTHWELL N. J., STOCK M. J., 1983. Acute effects of fat and carbohydrate on metabolic rate in normal, cold-acclimated and lean and obese (fa/fa) Zucker rats. Metabolism, 32, 371-376.

TRAYHURN P., 1979. Thermoregulation in the diabetic-obese $(\mathrm{db} / \mathrm{db})$ mouse. The role of non shivering thermogenesis in energy balance. Pflügers Arch., 380, 227-232.

TRAYHURN P., THURLBY P. L., JAMES W. P. T., 1973. A defective response to cold in the obese (ob/ob) mouse and the obese Zucker (fa/fa) rat. Proc. Nutr. Soc., 35, 133A.

TRIANDAFILLOU J., HIMMS-HAGEN J., 1983. Brown adipose tissue in genetically obese (fa/fa) rats : response to cold and diet. Am. J. Physiol., 244, E 145-E 150.

WICKLER S. J., HORWITZ B. A., STERN J. S., 1982. Regional blood flow in genetically obese rats during non shivering thermogenesis. Int. J. Obesity, 6, 481-490. 